\title{
Erratum to: New Spain: France Transmission Line: A Cost-Benefit Analysis
}

\author{
Stefan Perras · Friedrich Kunz • Dominik Möst
}

Published online: 7 May 2015

(C) Springer Fachmedien Wiesbaden 2015

Erratum to: Z Energiewirtsch (2015) 39:19-32

doi:10.1007/s12398-014-0144-x

Due to a processing error the title was incorrect. Please find the correct title below:

New Spain-France Transmission Line: A Cost-Benefit Analysis

The online version of the original article can be found under doi: http://dx.doi.org/10.1007/s12398-015-0151-6

S. Perras $(\bowtie) \cdot$ Prof. Dr. D. Möst

Fakultät Wirtschaftswissenschaften, Lehrstuhl für

Energiewirtschaft, Technische Universität Dresden,

01069 Dresden, Germany

e-mail: stefan.perras@mailbox.tu-dresden.de

Dr. F. Kunz

Deutsches Institut für Wirtschaftsforschung (DIW Berlin),

10117 Berlin, Germany 